\title{
Method for Measuring the Phase Spectrum of the Output of a Frequency Source Used in the Calibration of an Electroshock Weapon Characterization System
}

\author{
Nicholas G. Paulter, Jr. \\ National Institute of Standards and Technology, \\ Gaithersburg, MD 20899, USA \\ nicholas.paulter@nist.gov \\ Electroshock weapons (ESWs) output a transient burst of impulse-like high-voltage electrical signals that can incapacitate a human \\ target. To ensure safe and effective operation of ESW, their outputs must be accurately measured. Accordingly, the systems used to \\ measure the output of an ESW must be accurately characterized, and their response functions must be known for subsequent \\ processing of the ESW output waveforms (current and high voltage). The long epochs (> $5 \mathrm{~s}$ ) and high-bandwidth frequency content \\ (> $200 \mathrm{MHz}$ ) of the ESW output require unique measurement instrumentation and calibration tools. A frequency-domain swept- \\ frequency method is proposed here for the calibration of the phase response of an ESW measurement system. This method, although \\ motivated by the ESW measurement application, is also applicable to other systems.
}

Key words: calibration; magnitude response; phase calibration; phase response; signal source; swept frequency; waveform recorder.

Accepted: September 7, 2017

Published: September 19, 2017

https://doi.org/10.6028/jres.122.035

\section{Introduction}

An electroshock weapon (ESW) [1] provides a high-voltage (up to $5 \mathrm{kV}$ or more, depending on the ESW model, into an open circuit), low-current ( $<2$ A into an electrical load of about $500 \Omega$ ) output that can incapacitate a human target. Although there are different types of ESW configurations [1], this document is concerned only with those ESW that have at least two barbed electrodes that are launched from the ESW, travel a short distance to the target, and subsequently penetrate the skin of or attach to that target. An ESW, for the purpose here, is not a "stun gun" (or contact ESW as defined in Ref. [1]), which does not launch barbed electrodes. The use of EWSs is gaining popularity with law enforcement, security, and military agencies worldwide, and there are currently several ESW manufacturers worldwide.

The phase spectrum of the ESW output is important because it defines, along with the magnitude spectrum, the amplitude and transition durations of the output pulses, which may have consequences on the efficacy and safe use of ESWs (both of which are not the topic of this paper). The National Institute of Standards and Technology (NIST) has an ESW measurement system, the calibration of which is based on a hybrid of time-domain methods for the step/impulse response and swept-frequency methods for the magnitude spectrum. A missing component is a complementary swept-frequency method for measuring the phase spectrum. The phase measurement method presented here is applicable not only to ESW measurement systems and their calibration tools, but to other systems as well.

The ESW measurement system developed at NIST comprises a high-voltage probe; a current sensor; a customized, long-epoch (12 s), high-analog-bandwidth (200 MHz), 109 samples/second (S/s) waveform 
recorder; and associated support accessories (cables, connectors, etc.). To acquire both high-voltage and current waveforms simultaneously, a two-channel waveform recorder is required. The calibration of the NIST ESW measurement system is routinely performed using both step-like signals (the derivative of which are impulse-like signals), where these signals have been characterized by a calibrated reference oscilloscope, and swept-frequency methods. The term calibrated is being used in the sense of the International Vocabulary of Metrology (VIM) [2] definition: "operation that, under specified conditions, in a first step, establishes a relation between the quantity values with measurement uncertainties provided by measurement standards and corresponding indications with associated measurement uncertainties and, in a second step, uses this information to establish a relation for obtaining a measurement result from an indication.” In this case, the quantity values are the current and high-voltage outputs delivered by the ESW, and the measurement standards are NIST-traceable calibrated power meters for the magnitude of the frequency response of the ESW measurement system and a NIST-traceable reference electrical pulse generator for the step response. However, the output of the NIST-traceable reference electrical pulse generator is ultimately traceable to power meters for its magnitude spectral output. The phase spectral output is obtained using mathematical operations on the magnitude spectrum that are valid if the instrument exhibits certain idealized behavior [3]. There are other techniques for calibrating waveform recorders, but these are usually directed at high-bandwidth equivalent-time sampling instruments and are not applicable or appropriate for the ESW measurement system, which uses real-time single-shot waveform recorders [4-7]. Even for these high-bandwidth techniques, swept-frequency methods are used as the reference for their magnitude response. As with the reference electrical pulse generator, determination of the phase responses of these high-bandwidth methods also requires simplifying assumptions [8, 9].

Swept-frequency calibration techniques are typically much slower to perform than impulse response (or step response) methods, but they provide more accurate spectral information than do time-domain techniques. For this application of ESW characterization, accuracy is the primary consideration for the design of the measurement system and its calibration methods. The level of accuracy and measurement uncertainty required for ESW measurements is currently based on the manufacturer-stated performance specifications of the ESW. However, as the medical research community starts to define exposure limits, which may be more exacting than manufacturer specifications, the accuracy and uncertainty requirements for ESW measurements may change. Consequently, to be in a position to support as-of-yet undefined accuracy and uncertainty requirements for the NIST ESW measurement system, the more rigorous and exacting measurement calibration approaches are sought, and these can be relaxed at a later date if deemed appropriate.

To obtain an accurate estimate of the current and high-voltage pulses delivered by the ESW to its target, the influences of the measurement system on the waveforms (measured data) must be measured and removed from these waveforms. These effects are typically removed through a process called deconvolution, which requires an accurate estimate of the transfer function of the measurement system. The frequency response is a complex-valued function that can also be presented in terms of its magnitude and phase. For deconvolution to provide useful results, the frequency response of the measurement system to its input must be linear, and this response must have achieved a static (steady) state. Deconvolution is an inverse convolution problem. In time-domain processing, the deconvolution requires many nested arithmetic steps. In the frequency domain, on the other hand, deconvolution is a division of the complex spectrum of the waveform by the transfer function of the measurement system (or, equivalently, a division of the magnitude spectrum of the waveform by the magnitude response of the measurement system and the subtraction of the phase response of the measurement instrument from the phase spectrum of the waveform). Because deconvolution is mathematically simpler and less prone to numerical errors using frequency-domain methods than using time-domain methods, the focus here will be on the frequencydomain method, and specifically on a method for measuring the phase spectrum, since there are methods currently available for measuring the magnitude spectrum. The magnitude and phase (or real and imaginary) parts of the spectrum of the response of an instrument or the output of a generator are related to its impulse response or impulse-like signal output by the Fourier transform.

To compute the phase spectrum of the ESW output, the phase response of the measurement system must be known. It is proposed that this phase response be obtained using swept-frequency techniques, similar to the methods used to obtain the magnitude response, i.e., methods which are based on the 
nominally single-tone sinusoidal outputs of synthesized frequency sources. Consequently, the very first step is to obtain the phase spectrum of the frequency source output that will be used to calibrate the phase response of the measurement system, which comprises a waveform recorder. The purpose of this manuscript is to describe a method for measuring the absolute ${ }^{1}$ (except for a phase offset) phase spectrum of the output of the frequency source, which is a single-port device, and not a comparative or relative phase of this output to another source, such as would be done with a phase angle meter or phase detector. The application to characterizing the phase response of the measurement system will also be described.

\section{Reference Phase Measurement}

The output of the frequency source can be described in terms of its magnitude and phase components, both of which are dependent on frequency. This output, $V_{\text {src,i }}$, can be written as

$$
V_{s r, i}=A_{s r c, i} \sin \left(\omega_{i} t+\phi_{s r c, i}\right),
$$

where $i$ is the frequency index, $A_{s r c, i}$ is the amplitude of the output signal, $t$ is time, $\omega_{i}$ is the angular frequency ( $\omega_{i}=2 \pi f_{s r c, i}$, where $f_{s r c, i}$ is the frequency set by the operator), and $\varphi_{s r c, i}$ is the phase of the output signal. Determination of $\varphi_{s r c, i}$ has not previously been performed because (a) measurement methods have not been available, and/or (b) simulations would not be accurate or possible because the relationships between $\varphi_{s r c, i}$ and $\varphi_{s r c, j}, i \neq j$, for a given source, are too difficult to determine from the design of the instrument (the synthesize source), and/or the resultant estimated phase would have an uncertainty that exceeds useful levels.

\subsection{Measurement Setup and Process}

The general setup that is under development for measuring $\varphi_{s r c, i}$ is shown in Fig. 1. The measurement flow is shown in Fig. 1 by the arrowed lines. The signal source provides both a sinusoidal output ("signal out" inside the box labeled "SIGNAL SOURCE" in Fig. 1), the phase of which is used to determine the ESW measurement system phase response, and a reference out ("ref out"), which is used for synchronizing the other components of the measurement system. The reference output of the signal source must be at a fixed frequency independent of the frequency of its output signal; otherwise, this measurement method will not work. However, this requirement is not an impediment, because most reference outputs of synthesized sources are set at a fixed frequency, often $10 \mathrm{MHz}$, and typically have a sinusoidal waveform or a repetitive rectangular wave. The repetition rate of the pulse train output from the delay generator must be synchronous with and the same as that of the reference output of the signal source. The delay generator provides an adjustable delay that is controlled by the operator. The pulse train output from the delay generator goes to the pulse selector, which serves to reduce the repetition rate of the pulse train by periodically blocking pulses. The repetition rate of the pulse selector is set by the operator to have an integer relationship with the frequency of the reference output from the signal source. The purpose of the pulse selector is to ensure that no more than one sample is acquired per cycle of a sine wave. This requirement is a consequence of the phase measurement being based on direct current (dc) or lowfrequency voltage measurements. The pulses output from the pulse selector are sent to the drive electronics, which are required to trigger a sampling device or act as a sampling device, depending on the sampling technology used. For example, the drive electronics may accept a square-wave input and output a shortduration impulse-like signal to drive a sampling diode for conventional electronic sampling or a laser diode for electro-optic or photoconductive sampling. The sampling device interacts with the sinusoidal signal propagating along a transmission line in the pass-through sampler, where it is terminated into the characteristic impedance of the transmission line (typically $50 \Omega$ for high-speed/high-frequency signals). The pass-through sampler allows connection between the source and the instrument under test while

\footnotetext{
${ }^{1}$ Phase is always relative, but absolute is meant here to indicate that the phase relationship between frequencies of the sinusoidal signals output from the source is fixed and reproducible.
} 


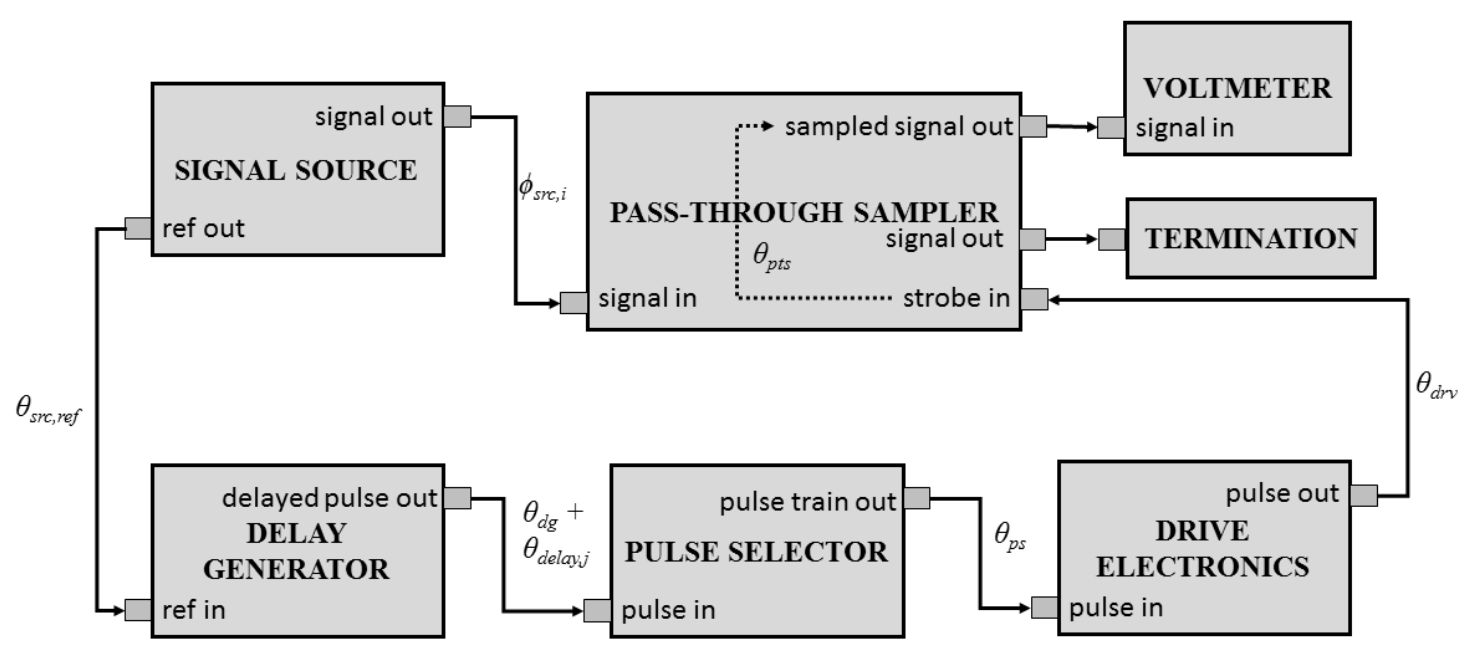

Fig. 1. Block diagram of a general system to measure the phase output from the signal source.

simultaneously measuring the phase output of the source. The input impedance of the termination is matched to the characteristic impedance of the transmission line, thus minimizing the magnitude of the reflections from the termination back to the transmission line. Because of the synchronization between the sampling instants and the signal source, the timing of the sampling instants can be set to specific instants (phases) of the sine wave through the adjustable delay. When the sampling device is activated, a sampled signal is produced. Each new setting of the delay results in a sampled signal that represents a different phase of the sine wave. After several delays, a set of values is obtained from which the phase of the sine wave is determined using conventional sine-wave fitting techniques. This is shown graphically in Fig. 2, in which a sine wave is depicted as being sampled at five delays (given by $d_{0}$ to $d_{4}$ ) to give the amplitude signals shown by the blue dots. The reference time is shown by the green line and is separate from the time delay. The reference time, $t_{0}$, must be the same for all frequencies measured; otherwise, a phase spectrum representative of the source cannot be obtained. The sine-wave fitting technique used in the simulations presented here is a three-parameter method, described in Institute of Electrical and Electronics Engineers (IEEE) Standard 1057 [10].

\subsection{Measurement Analysis}

The phase of the signal output from the signal source and measured on the transmission line of the pass-through sampler is given by:

$$
\phi_{c a l c, i}=\phi_{s c r . i}+\frac{1}{2} \theta_{p t s}-\left(\theta_{s r c, r e f}+\theta_{d g}+\theta_{d r v}+\theta_{p s}\right),
$$

where $\varphi_{s r c, i}$ is the phase of the source output signal; $\theta_{\text {src,ref }}$ is the phase delay between the reference output from the source and the reference input of the delay generator, which is constant; $\theta_{d g}$ is the phase delay between the pulse output of the delay generator and the pulse selector (its purpose is described in more detail in Sec. 2.3), which is constant; $\theta_{p s}$ is the phase delay between the pulse selector and the drive electronics, which is constant; $\theta_{d r v}$ is the phase delay between the drive electronics and the sampling gate, which is constant; $\theta_{p t s}$ is the propagation/phase delay as the output signal propagates along the transmission line (only half this phase value is used because the measurement is done in the middle of the transmission

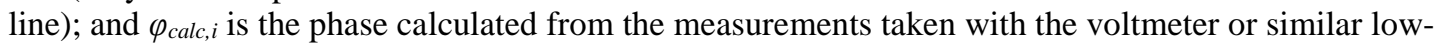
frequency instrument. In this method, $M$ measurements are taken at each of the $j$ delay settings, and this is done for each of the $i$ output frequencies from the source. In these simulations, $M$ was set to 1000 . 


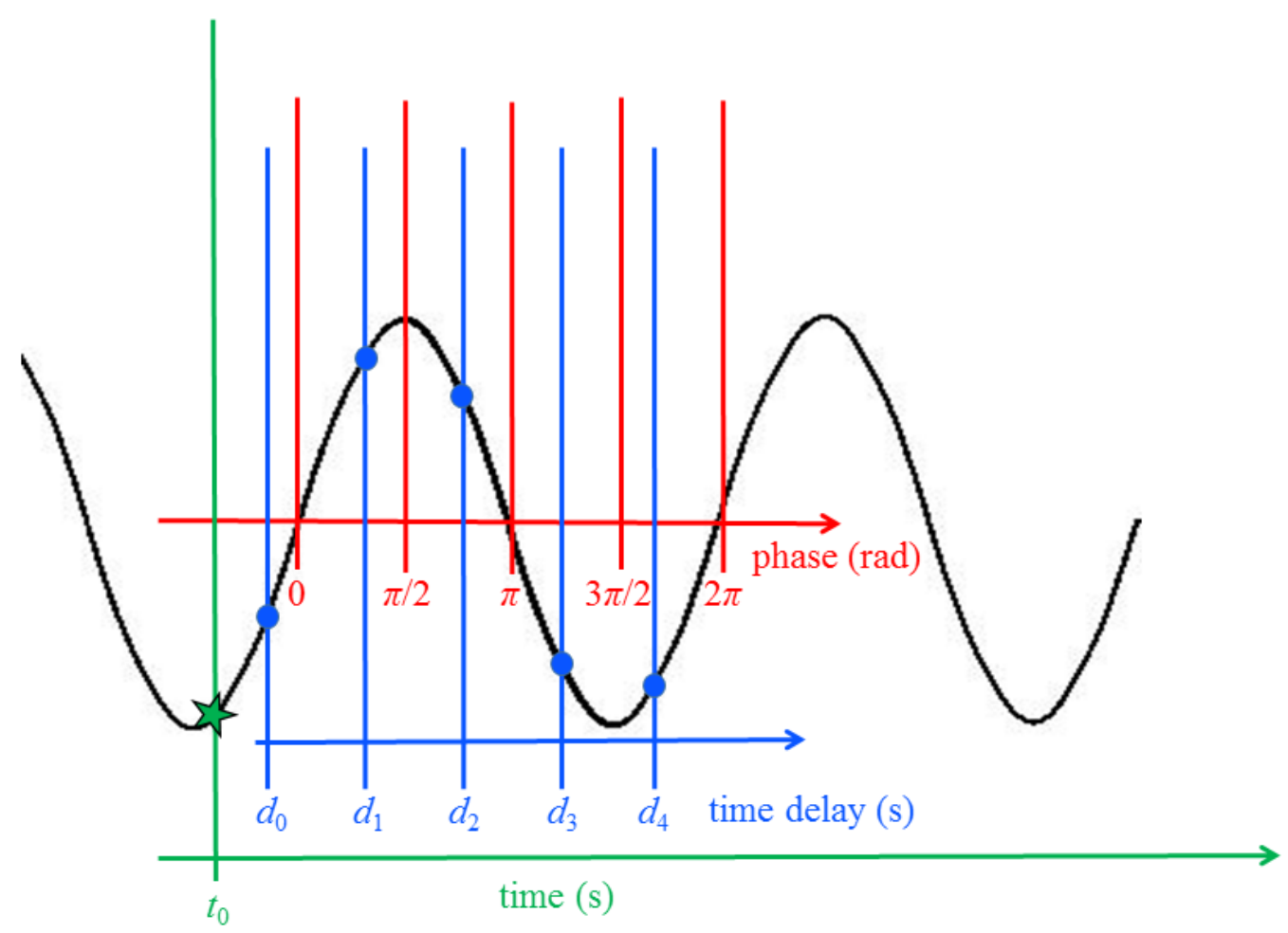

Fig. 2. Phase of the sinusoidal waveform relative to time delay settings and laboratory-defined time. The black line is a sinewave, the green coordinates represent the reference time axis, the blue coordinates represent the set time delays, and the red coordinates represent the phases of the sinewave.

Although more than one delay may be used per period of a sine wave, the case considered here is that when no more than one delay is used per period. More than one delay would be suitable for low-frequency signals, where one or fewer delays per period may necessitate a long time to acquire the data necessary for a phase measurement (see Sec. 2.3.2).

Figure 3 provides information on the fraction of the sine-wave period used to compute $\varphi_{\text {calc }, i}$. This figure shows the phase error as a function of the number of delays/period and of the fraction of the sinewave cycle. These results show that the period fraction should be close to one to minimize errors in $\varphi_{\text {calc, } i \text {. }}$ For signals without any additive noise (noise-free signals), there is no significant advantage in using more than three unique delay settings per period. However, numerical/computational noise and digitization noise still add to the signal. Figure 4 shows that shorter-duration integration intervals (or sampling apertures) produce less phase error than do the longer-duration integration intervals. To reduce the variance in the phase measurement, these data suggest that it is better to average many shorter-duration events than to average a few longer-duration events. Figure 5 shows that the addition of noise does not change the overall relationship between the phase error and the period fraction and the integration interval (compare to Fig. 4).

Figure 6 shows that there is a random association between phase error and phase offset. However, the more unique delays used per period, the lower is the phase error, and this relationship between phase error and delays/period exhibits a nominal $1 / \sqrt{N}$ behavior (where $N$ is the number of delays/period) (see Fig. 7).

\subsection{Measurement System Considerations}

There should be at least three unique delay values when using the sine-wave fitting technique [10] to compute each of the $\varphi_{\text {calc, }, \text {, and these delay values should span about one full period at each of the } i \text { th }}$ frequencies to be measured (see Figs. 3 and 4). From the measurements at these unique delay settings, a $\varphi_{\text {calc }, i}$ value is obtained (illustrated by the green star in Fig. 2). Each ith frequency results in a unique $\varphi_{\text {calc }, i}$ 


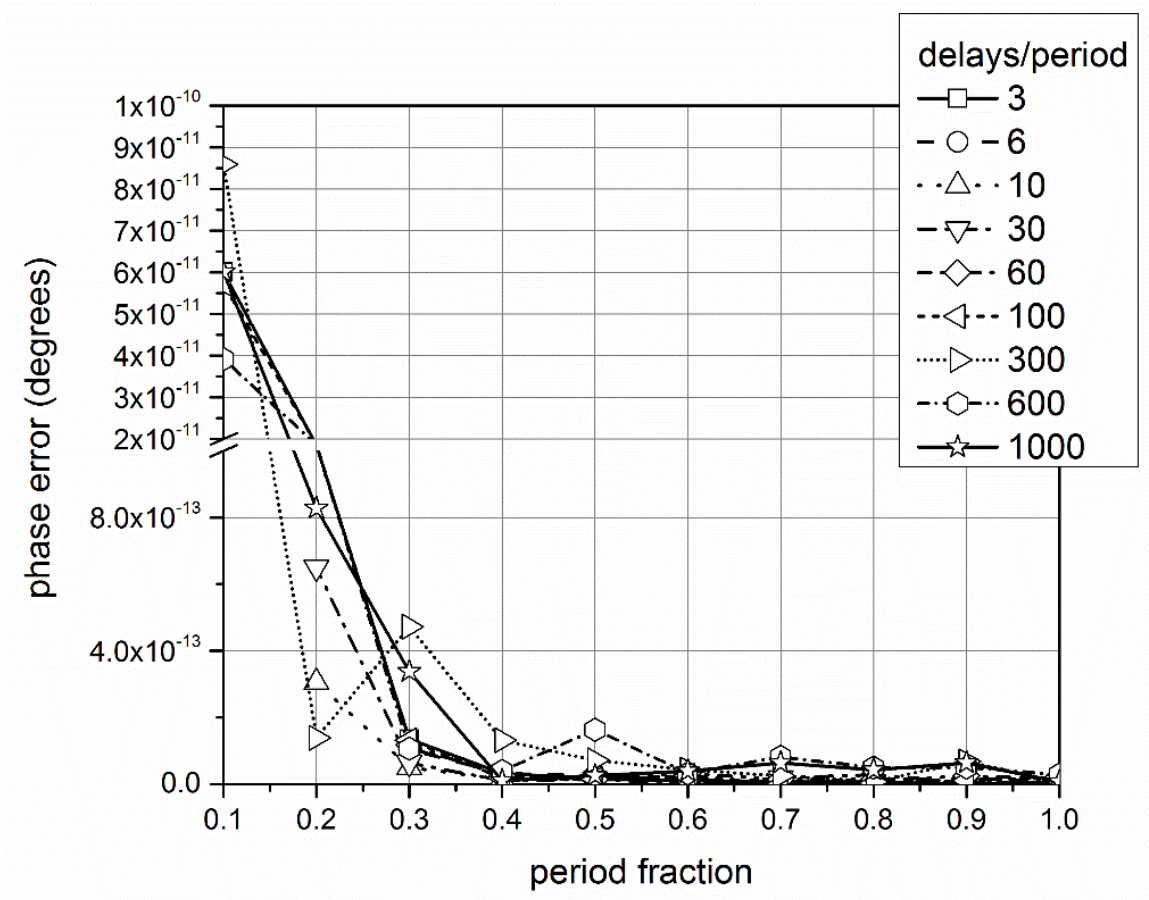

Fig. 3. Simulated phase error (degrees) for different delay settings and different fractions of a sinewave cycle. The root mean square (rms) noise was set to 0 , the phase offset was set to $25^{\circ}$, and 1000 samples were averaged for each datum shown.

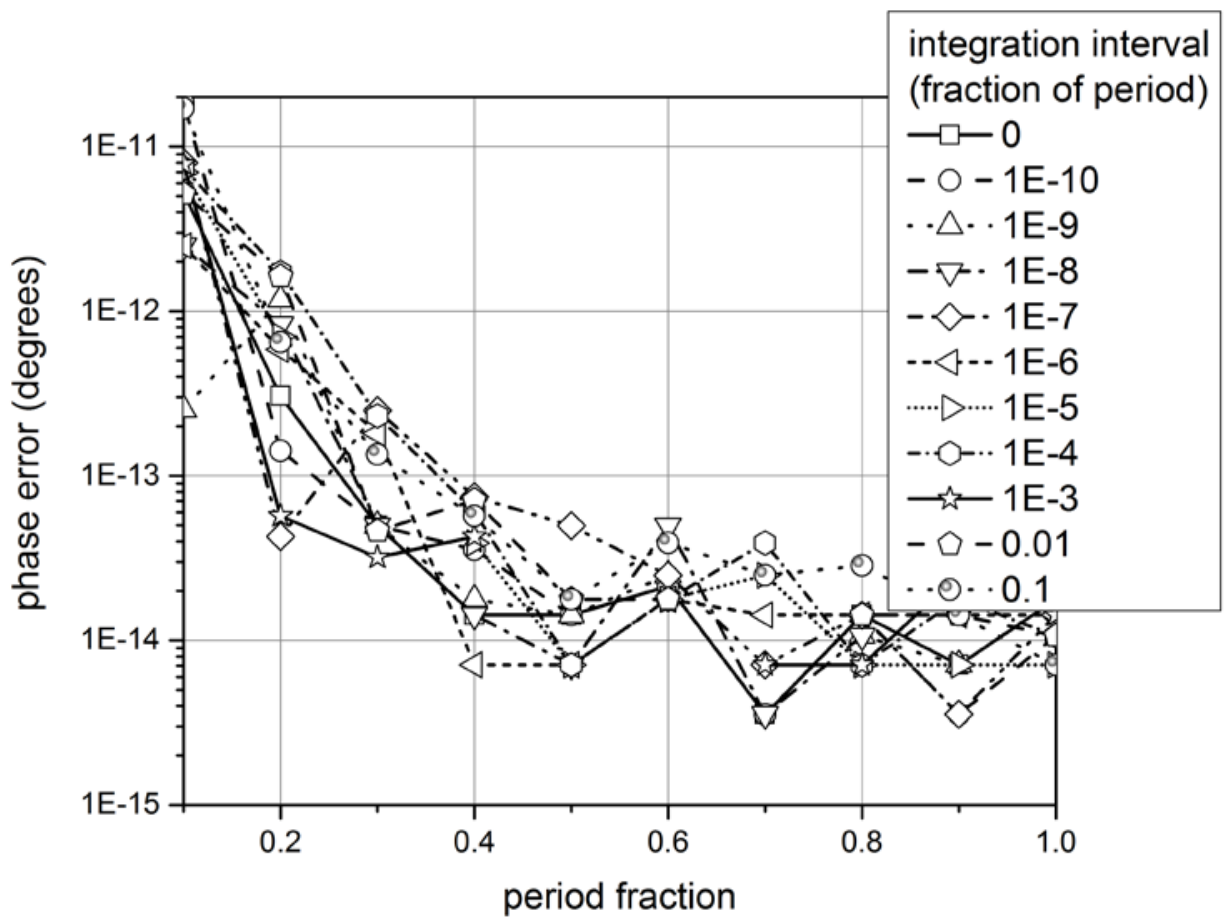

Fig. 4. Simulated phase error (degrees) for different integration intervals and different fractions of a sinewave cycle. The rms noise was set to 0 , the phase offset was set to $25^{\circ}$, and 1000 samples were averaged for each datum shown. 


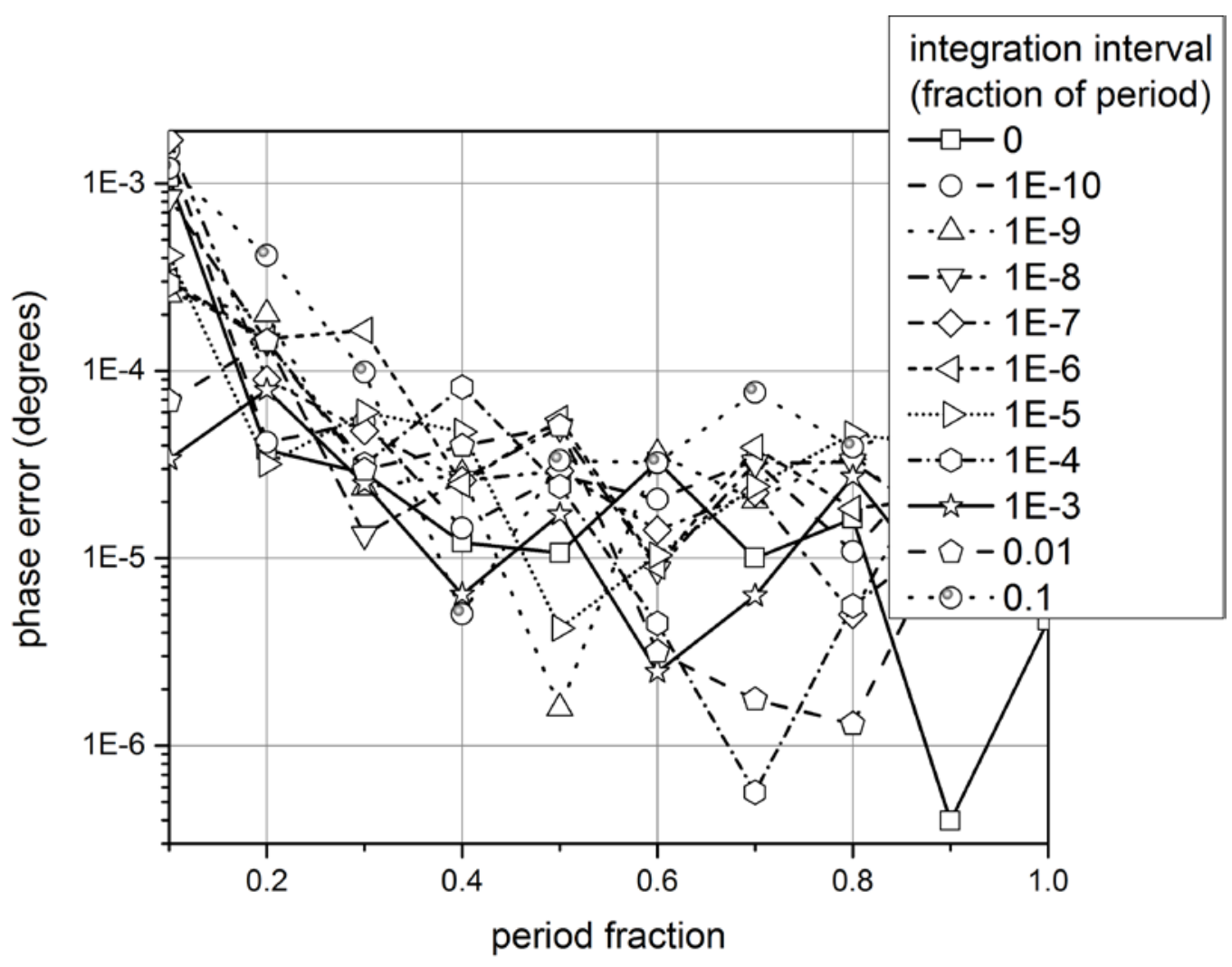

Fig. 5. Simulated phase error (degrees) for different integration intervals and different fractions of a sinewave cycle. The rms noise was set to $1 \times 10^{-6} A_{\text {src }}$, the phase offset was set to $25^{\circ}$, and 1000 samples were averaged for each datum shown.

value that, for the purpose of illustration, could be placed on the green line in Fig. 2 . This is the reason that the reference time must be the same for all measured frequencies. If this reference time is not maintained, $\varphi_{\text {calc }, i}$ will not be associated with $\varphi_{\text {src, }, \text {. }}$

This particular measurement system design allows the phase of $V_{\text {src }, i}$ to be measured from very low frequencies $(<<1 \mathrm{~Hz})$ to very high frequencies $(>>1 \mathrm{GHz})$, with, perhaps, only changes in the drive electronics or exchanges in the positions of the drive electronics and the delay generator. Regularly spaced sampling, such as many sample instants per cycle, would work with this design, but only for the lower frequency ranges and with the constraints that (a) the voltmeter (or similar low-frequency instrument for acquiring an average signal) has a sufficiently fast response that relaxes between successive sample instants, and (b) the delay generator can achieve successive delays rapidly to allow sampling at the appropriate sampling instants. As mentioned previously, this measurement system provides an absolute phase measurement, other than for an arbitrary phase offset, of the output phase spectrum of a sinusoidal signal source, which is a single-port device. Typical waveform sampling methods would not provide this information because the waveform recorder (sampling oscilloscope) may have a unique but unknown phase response for each frequency. Phase comparator/detection methods would not work because of the reliance on an external source for comparison, the unknown phase response of the phase detector circuit, and the broad frequency range required for calibration of the impulse response of an ESW measurement system. 


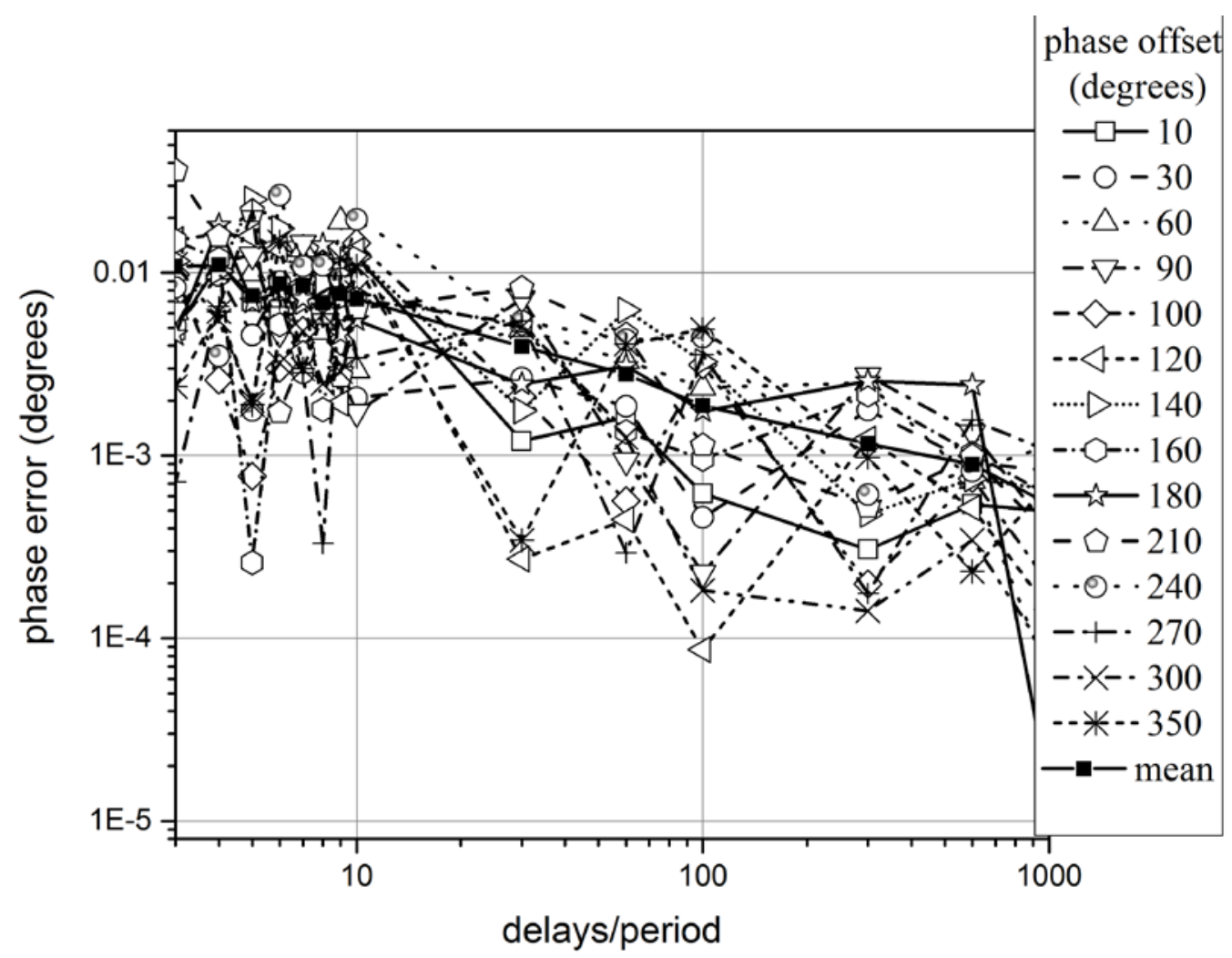

Fig. 6. Simulated phase error (degrees) for different delay settings and different phase offsets. The rms noise was $0.01 A_{\text {src }}$, and each value presents the average of 1000 events.

Understanding the purpose of the pulse selector is critical. The pulse selector ensures that only one sampling function occurs for each period of $V_{s r c, i}$. This can be best visualized by considering $V_{s r c, i}$ as a sine wave and the sampling function as a fine-toothed comb. No more than one tooth of the comb should be registered with each period of the sine wave (see Fig. 8). This one tooth can then be synchronized with any phase on the sine wave through the delay generator. Each output frequency of the signal source has a different set of delays and possibly a different comb frequency through the pulse selector. If the repetition rate of the delay generator output changes for any $V_{s r c, i}$, it may not be possible to compute a phase response for the source, because (a) the phase relationships amongst the repetition rates may be too difficult to correct based on the knowledge of the electrical design of the delay generator, and/or (b) the phase uncertainty that is calculated based on this design would exceed useful levels. To satisfy the requirement that only one unique phase per period of the sine wave is measured per delay setting, where the delay generator repetition rate is fixed, the sampling repetition rate must also be fixed. This is accomplished by periodically blocking pulses produced by the pulse selector.

Once the measurement process is repeated for a set of frequencies, the result is a set of $\varphi_{c a l c, i}$ values, which represents the phase spectrum of the frequency source output. Each of these $\varphi_{\text {calc, } i \text { values contains all }}$ the static phase contributions shown in Eq. (2), and they are related by a common phase offset, which can be arbitrarily defined as the phase of the lowest frequency component and subtracted from all phase values. This phase measurement method provides phase values less integer multiples of $2 \pi \mathrm{rad}$, which is also how the phase response of the ESW measurement system and the phase spectrum of the ESW output are presented. 


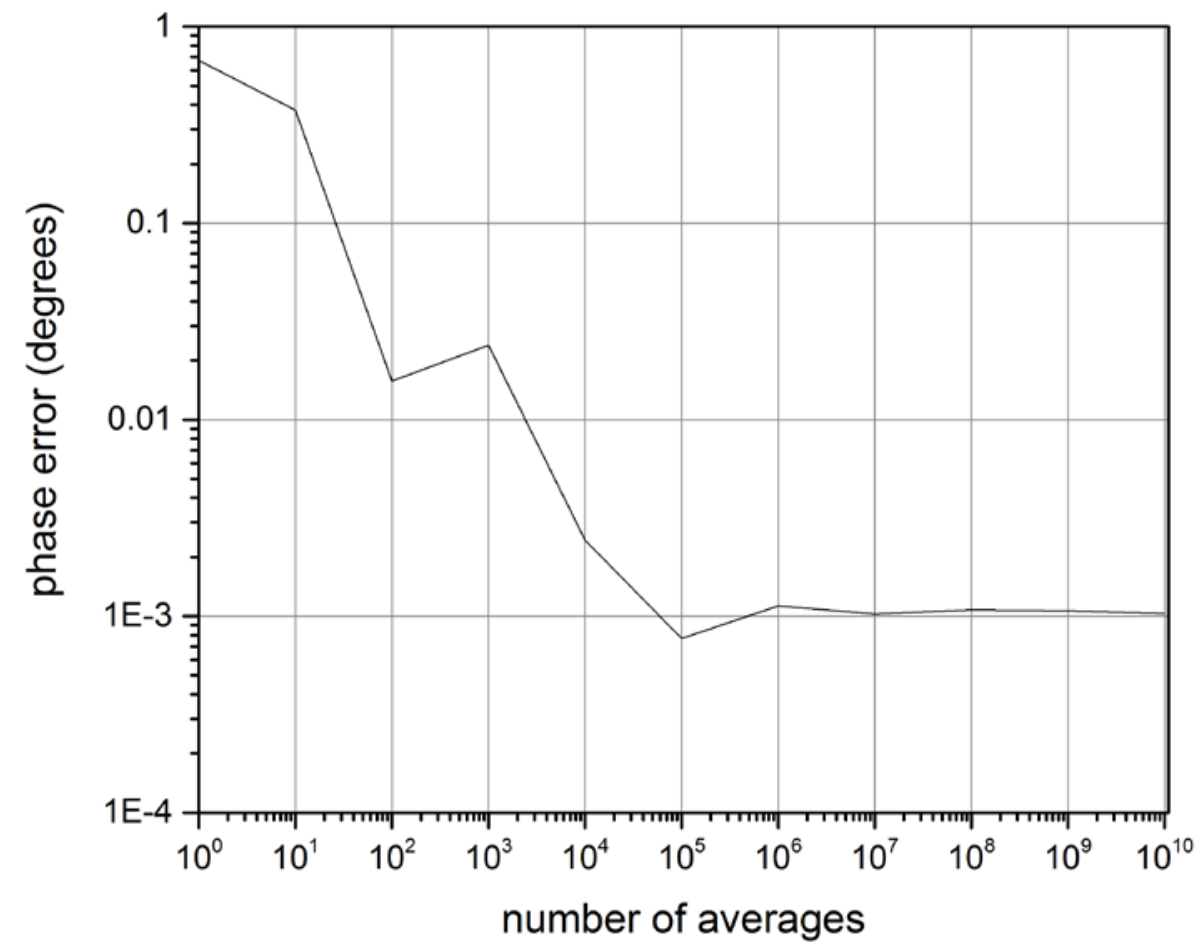

Fig. 7. Simulated phase error (degrees) as a function of the number of averages. The rms noise was $0.01 A_{\text {src }}$, the phase offset was $25^{\circ}$, and 3 delays/cycle were used.

Operational considerations when implementing this measurement method are:

- Jitter and noise affect the upper limit of the measurable frequency range (see Sec. 2.3.2).

- Measurement time limits the low-frequency range (see Sec. 2.3.2). The lower frequency limit is primarily impacted by the duration of the measurement process.

- The repetition rate of the delay generator must not change for the set of $V_{s r c, i}$ values. If it does change, then this change may introduce a repetition-rate-dependent component to $\theta_{d g}$, which will prevent construction of a phase spectrum with appropriately associated phase components.

- The pulse selector must not allow more than one sampling pulse to transmit for each period of the sine wave (except, perhaps, for the case of low-frequency signals, as previously discussed). If this requirement is not met, the $\varphi_{c a l c, i}$ cannot be found that can be used to compute the phase spectrum of the source output.

- The $\varphi_{s r c, i}$ is stable and reproducible with respect to $\theta_{\text {src,ref }}$ for each $i$ th frequency.

- The phase response of the waveform recorder under test is stable and reproducible for each unique timebase setting.

\subsubsection{Pulse Selection}

The criterion for the pulse selector is that it allows only one $\varphi_{\text {calc,i }}$ to be measured per period of a given sine wave and for a given delay, $j$. However, this $\varphi_{\text {calc }, i}$ can be the result of the average of $M$ cycles simultaneously because of the synchronization of the repetitive source signal and the repetitive sampling events (see Fig. 8). The repetition rate of the output of the pulse selector is adjusted to provide no more than one sampling event per period of the source output, and this relationship can be described by a pulse selection ratio, $r_{p s}$, which is given by 


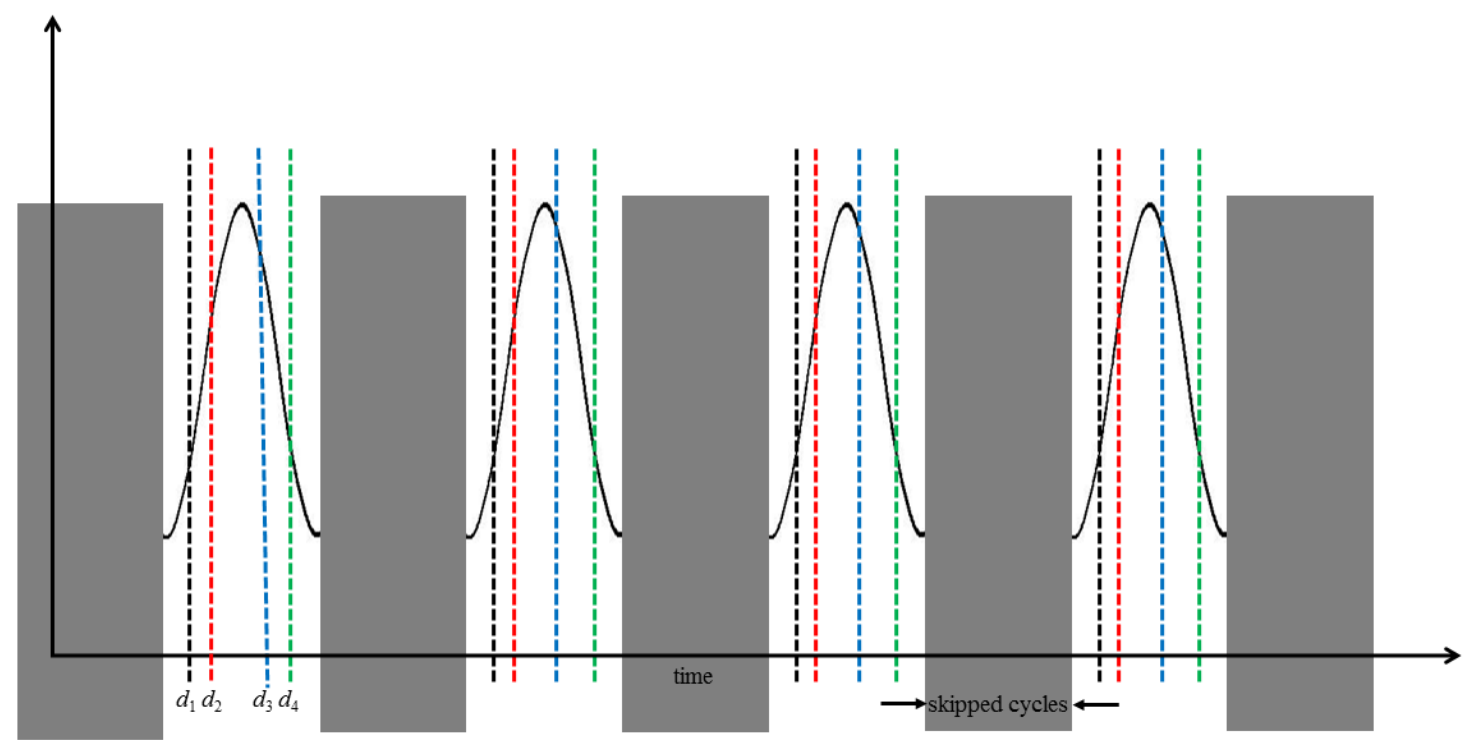

Fig. 8. A sinewave showing four different delays (each depicted as a different color) at which the sinewave is measured. Each delay corresponds to a unique time (phase) of the sinewave. The phase measurements are done consecutively: the delay is set to $d_{1}$, and the phase is measured after averaging over $M$ cycles, the delay is then set to $d_{2}$, and the phase is measured over $M$ cycles, and similarly for delays $d_{3}$ and $d_{4}$. The periodicity of a given delay describes a comb of measurement times that are synchronized with the output signal. The shaded block given as skipped cycles represents the cycles that may be skipped for synchronization between the source and sampling frequencies.

$$
r_{p s}=\frac{f_{r e f}}{\operatorname{GCF}\left(f_{s r c}, f_{r e f}\right)},
$$

where $G C F\left(f_{\text {src }}, f_{r e f}\right)$ is the greatest common factor of $f_{s r c}$ and $f_{r e f}$, and $f_{\text {ref }}$ is the frequency of the reference signal of the source. Before setting $r_{p s}, f_{s r c}$ and $f_{\text {ref }}$ should be accurately known. If they are not, these frequency errors will contribute to phase measurement errors. However, four-parameter sine-fit methods can be used if the frequency is an unknown. According to Ref. [10], "Even if the input frequency is accurately known a priori, the four-parameter method usually determines it to even better accuracy."

In some cases, it may be necessary to sample a phase less than once per period. The ratio, $r_{\text {skip }}$, of skipped cycles to the source frequency can be computed using the common factors of $f_{\text {src }}$ and $f_{\text {ref }}$. The $r_{\text {skip }}$ ratio is not a settable value; it is an indicator of the number of periods of the signal source that are not measured, and so it provides a measure of the efficiency of the measurement process. The highest efficiency is for $r_{s k i p}=0$, and the efficiency decreases for increasing values of $r_{\text {skip }}$. The values of $r_{p s}, f_{p s}$, and $r_{\text {skip }}$ can be found by the following steps:

(1) determine the common factors for the pair of $f_{s r c}$ and $f_{r e f}$,

(2) compute $r_{p s}$,

(3) set $f_{p s}=G C F\left(f_{s r c}, f_{r e f}\right)$, and

(4) divide $f_{\text {src }}$ by the $G C F\left(f_{\text {src }}, f_{\text {ref }}\right)$ and subtract 1 from this to obtain $r_{\text {skip. }}$.

The pulse selector is set to provide an output pulse train with a repetition rate of $f_{p s}$. Table 1 shows some example values of $f_{p s}, r_{p s}$, and $r_{s k i p}$ for given values of $f_{s r c}$, using $f_{r e f}=10 \mathrm{MHz}$ (a common reference).

The uncertainty, $u_{r_{p s}}$, in $r_{p s}$, can be described by: 


$$
u_{r_{p s}}=\frac{f_{r e f}}{G C F} \sqrt{\left(\frac{u_{f_{r e f}}}{f_{r e f}}\right)^{2}+\left(\frac{u_{G C F}}{G C F}\right)^{2}},
$$

where $u_{f_{r e f}}$ is the uncertainty in the reference frequency, and $u_{G C F}$ is the uncertainty in GCF. The GCF can vary significantly depending on the values of $f_{\text {src }}$ and $f_{\text {ref. }}$. For example, if $f_{\text {src }}=507000 \mathrm{~Hz}$, and $f_{\text {ref }}=10000$ $000 \mathrm{~Hz}$, then $G C F=1000$. If $f_{s r c}$ is changed to $507010 \mathrm{~Hz}$, for the same $f_{r e f}, G C F=10$. The $u_{G C F}$ can also vary significantly depending on $f_{s r c}$ and $f_{\text {ref }}$ and their uncertainties. Table 2 provides estimates for $u_{G C F}$ with a fixed $f_{\text {ref }}$ and for various tolerances on $f_{\text {src }}$. Because $f_{\text {src }}$ and $f_{\text {ref }}$ will not vary independently, because they are coupled by design of the measurement system (see Fig. 1), the $u_{G C F}$ values presented in Table 2 are worst-case estimates and are based on the ability to resolve the values of $f_{s r c}$ and $f_{\text {ref. }}$. Nevertheless, $f_{s r c}$ and $f_{r e f}$ and their associated frequency uncertainties should be determined, and $u_{G C F}$ should be computed from this information. An erroneous value of GCF will result in an incorrect computation of $r_{p s}$ and $r_{s k i p}$, and this will cause the average of the measured amplitude values at $\varphi_{s r c, i}$ to approach zero for any delay setting. Therefore, $r_{p s}$ and $r_{\text {skip }}$ do not affect the values of $\varphi_{\text {src,i }}$, but they do affect the efficient acquisition of the amplitude values used in its computation.

Table 1. Values of $f_{p s}, r_{p s}$, and $r_{\text {skip }}$ for given values of $f_{\text {src }}$, using $f_{\text {ref }}=10 \mathrm{MHz}$.

\begin{tabular}{|c|c|c|c|c|c|}
\hline$f_{\text {src }}(\mathrm{Hz})$ & $f_{\text {ref }}(\mathrm{Hz})$ & $G C F\left(f_{\text {src }}, f_{\text {ref }}\right)$ & $r_{p s}$ & $f_{p s}(\mathrm{~Hz})$ & $r_{\text {skip }}$ \\
\hline 1 & 10000000 & 1 & 10000000 & 1 & 0 \\
\hline 4 & 10000000 & 4 & 2500000 & 4 & 0 \\
\hline 23 & 10000000 & 1 & 10000000 & 1 & 22 \\
\hline 100 & 10000000 & 100 & 100000 & 100 & 0 \\
\hline 120 & 10000000 & 40 & 250000 & 40 & 2 \\
\hline 1000 & 10000000 & 1000 & 10000 & 1000 & 0 \\
\hline 6500 & 10000000 & 500 & 20000 & 500 & 12 \\
\hline 10000 & 10000000 & 10000 & 1000 & 10000 & 0 \\
\hline 33000 & 10000000 & 1000 & 10000 & 1000 & 32 \\
\hline 100000 & 10000000 & 100000 & 100 & 100000 & 0 \\
\hline 507000 & 10000000 & 1000 & 10000 & 1000 & 506 \\
\hline 876000 & 10000000 & 4000 & 2500 & 4000 & 218 \\
\hline 1000000 & 10000000 & 1000000 & 10 & 1000000 & 0 \\
\hline 1100101 & 10000000 & 1 & 10000000 & 1 & 1100100 \\
\hline 5403000 & 10000000 & 1000 & 10000 & 1000 & 5402 \\
\hline 10000000 & 10000000 & 10000000 & 1 & 10000000 & 0 \\
\hline 35000000 & 10000000 & 5000000 & 2 & 5000000 & 6 \\
\hline 120000000 & 10000000 & 10000000 & 1 & 10000000 & 11 \\
\hline 500000010 & 10000000 & 10 & 1000000 & 10 & 50000000 \\
\hline
\end{tabular}

Table 2. Estimates of $u_{\mathrm{GCF}}$, with $f_{\text {ref }}=10 \mathrm{MHz}$, and for various tolerances on the value of $f_{\text {src }}$.

\begin{tabular}{|c|c|c|}
\hline$f_{s r c}(\mathrm{~Hz}) ; G C F^{\mathrm{a}}$ & $f_{\text {src }}$ tolerance $(\mathrm{ppm})$ & $u_{G C F}$ \\
\hline \multirow{4}{*}{$6500 ; 500$} & 1000 & 132 \\
\hline & 100 & 210 \\
\hline & 10 & 0 \\
\hline & 1 & 0 \\
\hline \multirow{4}{*}{507 000; 1000} & 1000 & 107 \\
\hline & 100 & 96 \\
\hline & 10 & 298 \\
\hline & 1 & 113 \\
\hline \multirow{4}{*}{5403 000; 1000} & 1000 & 50 \\
\hline & 100 & 146 \\
\hline & 10 & 96 \\
\hline & 1 & 290 \\
\hline \multirow{4}{*}{120000 000; 10000000} & 1000 & 316216 \\
\hline & 100 & 316226 \\
\hline & 10 & 316227 \\
\hline & 1 & 705689 \\
\hline
\end{tabular}




\subsubsection{Measurement Time and Jitter}

Another consideration when implementing this measurement method is the time for making the phase measurements of low frequencies. Although there is no low-frequency measurement limit, there is a practical limit to the time required to perform the measurement. The measurement time, $T_{m}$, (in units of s/measurement), can be expressed as:

$$
T_{m}=\frac{K_{\text {pulses }}}{\text { delay }} \frac{L_{\text {delays }}}{\text { period }} \frac{N_{\text {per }}}{\text { measurement }} \frac{T_{p s}}{\text { pulse }},
$$

where $K_{\text {pulses }}$ is the number of pulses that is averaged at each $j$ th delay setting (which is a user-defined value), $L_{\text {delays }}$ is the number of delays used per period of $f_{\text {src }}$ (where $L$ is a user-defined integer), $N_{\text {per }}$ is the number of periods needed to perform a measurement $\left(N_{p e r}=r_{\text {skip }}+1\right)$, and $T_{p s}$ is the delay between pulses $\left(T_{p s}=1 / f_{p s}\right)$. Table 3 provides information on possible measurement times.

Table 3. Measurement time, $T_{m}$, as a function of various measurement parameters.

\begin{tabular}{|c|c|c|c|c|c|}
\hline$T_{m}$ (s/measurement) & $f_{\text {src }}$ & $K_{\text {pulses }}$ & $L_{\text {delays }}$ & $N_{\text {per }}$ & $T_{p s}(\mathrm{~s})$ \\
\hline 5000 & 1 & 1000 & 5 & 1 & 1 \\
\hline 375 & 120 & 1000 & 5 & 3 & $1 / 40$ \\
\hline 165 & 33000 & 1000 & 5 & 33 & $1 / 1000$ \\
\hline 0.005 & 1000000 & 1000 & 5 & 1 & $1 / 1000000$ \\
\hline 0.0005 & 10000000 & 1000 & 5 & 1 & $1 / 10000000$ \\
\hline 0.007 & 35000000 & 1000 & 5 & 5 & $1 / 5000000$ \\
\hline 25000000500 & 500000010 & 1000 & 5 & 50000001 & $1 / 10$ \\
\hline
\end{tabular}

Based on this formula, quite a large range of measurement times may be necessary. However, certain extremely long times can be avoided, and others can be reduced. For example, the last line of Table 3 shows a measurement time of almost $800 \mathrm{yr}$. Upon examination, measuring the phase with a $10 \mathrm{~Hz}$ offset at $f_{s r c} \approx 500 \mathrm{MHz}$ may not be realistic, as this lower-frequency sideband is at $2 \times 10^{-8}$ of $f_{\text {src }}$. Most circuits are not going to exhibit or demonstrate a significantly different response at these two frequencies. It may be more realistic to consider frequencies within about $0.0001 f_{s c c}$, or in this case, for $f_{s r c}=500100000 \mathrm{~Hz}$. For an $f_{s r c}=500100000 \mathrm{~Hz}$ signal, $T_{m}$ would be about $250 \mathrm{~s}$ (a 5000-fold reduction in $T_{m}$ compared to the case with $f_{s r c}=500000010 \mathrm{~Hz}$ ). Also, the value of $K_{\text {pulses }}$ given in the table may be too high. Because a sine-fit algorithm is used, which is somewhat insensitive to noise (see Fig. 5), $K_{\text {pulses }}<100$ may be more realistic. Furthermore, the duration of the sampling function also affects the signal-to-noise ratio (see Fig. 4), so that further reduction in measurement time may be achieved with shorter-duration sampling functions.

For the upper frequency limit, jitter effectively acts as a low-pass filter, thus reducing the amplitude of the acquired sine wave. If this reduction causes the sine-wave amplitude to be less than the noise level of the waveform recorder, then this method may not work without further modifications. Based on our observations of the performance of typical waveform recorders, the method we described can easily be used to $20 \mathrm{GHz}$, well beyond the current requirements for ESW characterization. Jitter may also bias the signal value that is used to represent the signal value at the nominal delay setting. This bias will depend on the slope of the sine wave at the sampling instant and the shape of the sampling aperture.

\section{Phase Measurement of the Waveform Recorder}

The measurement of the phase response of the waveform recorder is discussed in this section. When acquiring the sinusoidal signals from the waveform recorder, the phase of the sine wave as measured by the waveform recorder can be written as:

$$
\phi_{\text {meas }, i}=\phi_{s r c, i}+\theta_{s r c, 0}+\phi_{w r, i},
$$


where $\theta_{s r c, 0}$ is the phase delay between the reference output from the source and the reference input of the waveform recorder, which is constant; $\varphi_{w r, i}$ is the phase response of the waveform recorder, which is the sought after value; and $\varphi_{\text {meas, } i}$ is the phase as exhibited in the waveform recorder data. Equation (4) can be rearranged to solve for $\varphi_{w r, i}$, and, by making a substitution for $\varphi_{s r c, i}$ based on the solution from Eq. (2), this gives:

$$
\begin{aligned}
\phi_{w r, i} & =\phi_{\text {meas }, i}-\theta_{\text {src }, 0}-\phi_{s r c, i} \\
& =\phi_{\text {meas }, i}-\theta_{\text {sr }, 0}-\phi_{c a l c, i}+\theta_{\text {sr }, \text { ref }}+\theta_{d g}+\theta_{d r v}+\frac{1}{2} \theta_{T L} \\
& =\phi_{\text {meas }, i}-\phi_{c a l c, i}+\theta_{\text {off }},
\end{aligned}
$$

where $\theta_{\text {off }}$ is the sum of all the constant phase terms. The uncertainty, $u_{\varphi_{w r, i}}$, in $\varphi_{w r, i}$ is given by:

$$
u_{\varphi_{w r, i}}=\sqrt{u_{\varphi_{\text {meas }, i}}^{2}+u_{\varphi_{\text {cac }, i}}^{2}+u_{\theta_{\text {off }}}^{2}},
$$

where $u_{\varphi_{\text {meas }, i}}, u_{\varphi_{\text {calc }, i}}$, and $u_{\theta_{\text {off }}}$ represent the uncertainties for $\varphi_{\text {meas }, i}, \varphi_{\text {src }, i}$, and $\theta_{\text {off. }}$ The value of $\theta_{\text {off }}$ is not important because it is a constant and, in the time domain, represents a lossless time delay. Furthermore, because phase is a relative value, the value of $\theta_{\text {off }}$ can be subtracted without changing the phase response of the waveform recorder as measured for a given frequency band. Although detailed measurements have not yet been completed, preliminary data show an improvement (reduction) in the uncertainty of $\varphi_{s r c, i}$ of over 100 times that of current methods for the frequency range of millihertz to megahertz. If we estimate that $u_{\varphi_{\text {meas }, i}} \approx u_{\varphi_{\text {cal }, i}}$ and $u_{\theta_{\text {off }}}$ can be ignored, a possible reduction in $u_{\varphi_{w r, i}}$ of more than $100 / \sqrt{2}$ can be expected.

\section{Summary}

A frequency-domain swept-frequency method is proposed here for the measurement of the phase of the output of a sinusoidal signal source. This method is unique in that it provides an absolute phase spectrum of the signal source output and not simply a relative phase value at a specific frequency. It has the potential for significant reduction in phase measurement uncertainty compared to conventional methods. The results of this phase measurement, in conjunction with a conventional swept-frequency method for measuring the magnitude of the signal source output, provide a method for capturing the phase and magnitude spectrum of the output of the frequency source. This spectrum can subsequently be used to characterize the complex frequency response of a waveform recorder, specifically here with an application for an ESW measurement system.

\section{Acknowledgments}

The author would like to acknowledge Donald Larson, Sergio Rapuano, and Francesco Picariello for detailed technical discussions and Erik Timpson for a thorough technical review and comments.

\section{References}

[1] International Electrotechnical Commission (IEC) (2015) IEC 62792:2015-Measurement method for the output of electroshock weapons (IEC, Geneva, Switzerland).

[2] Joint Committee for Guides in Metrology (JCGM) (2012) 200:2012-International vocabulary of metrology-Basic and general concepts and associated terms (VIM). http://www.iso.org/sites/JCGM/JCGM-introduction.htm

[3] Oppenheim AV, Schafer RW (2009) Discrete-time Signal Processing (Prentice Hall, Englewood Cliffs, NJ), 3rd Ed.

[4] Gans W L (1990) Dynamic calibration of waveform recorders and oscilloscopes using pulse standards. IEEE Transactions on Instrumentation and Measurement 39(6):952-957. https://doi.org/10.1109/IMTC.1990.66010 
[5] Clement TS, Hale PD, Williams DF, Wang CM, Dienstfrey A, Keenan DA (2006) Calibration of sampling oscilloscopes with high-speed photodiodes. IEEE Transactions on Microwave Theory and Techniques, 54(8):3173-3181. https://doi.org/10.1109/TMTT.2006.879135

[6] Larson DR, Paulter NG (2001) Using the nose-to-nose sampler calibration method in pulse metrology. Proceedings of 11th IMEKO TC-4 Symposium, pp 461-465.

[7] Fuser H, Eichstadt S, Baaske K, Elster C, Kuhlmann K, Judaschke R, Pierz K, Bieler M (2012) Optoelectronic time-domain characterization of a $100 \mathrm{GHz}$ sampling oscilloscope. Journal of Measurement Science and Technology 23(2):025201. https://doi.org/10.1088/0957-0233/23/2/025201

[8] Hale PD, Clement TS, Coakley KJ, Wang CM, DeGroot DC, Verdoni AP (2000) Estimating the magnitude and phase response of a $50 \mathrm{GHz}$ sampling oscilloscope using the nose-to-nose method. Proceedings of IEEE 55th ARFTG Conference DigestSpring, pp 1-8. https://doi.org/10.1109/ARFTG.2000.327397

[9] Paulter NG, Larson DR (2007) Uncertainty in the nose-to-nose sampler calibration method due to the assertion that generator and receiver functions are identical. IEEE Transactions on Instrumentation and Measurement 56(6):2570-2576. https://doi.org/10.1109/TIM.2007.908134

[10] Institute of Electrical and Electronics Engineers (IEEE) 1057-2007 IEEE Standard for Digitizing Waveform Recorders (IEEE, New York, NY). http://ieeexplore.ieee.org/servlet/opac?punumber=5981349

About the author: Nicholas G. Paulter, Jr. is a group leader in the Materials Measurement Science Division at NIST. The group he leads conducts research and develops and disseminates the measurement science to support the development of performance-based standards for and promote the advancement of the technologies used in trauma-mitigating materials and material systems, the sensing of concealed threats and contraband, advanced composites, high-performance materials, safety and security applications, and forensics.

The National Institute of Standards and Technology is an agency of the U.S. Department of Commerce. 